\title{
Tratamiento endoscópico de complicaciones de cirugía bariátrica
}

\author{
Carlos Valenzuela-Salazar* \\ Hospital General Dr. Manuel Gea González, Ciudad de México, Méx., México
}

\begin{abstract}
Resumen
Si bien la cirugía bariátrica tiene baja morbimortalidad, los procedimientos realizados mundialmente se han incrementado exponencialmente y con eso el número total de complicaciones entre las que destacan las fugas o fístulas por su alta morbimortalidad, así como las estenosis y hemorragia por su relativa alta frecuencia. Para fístulas existe mayor nivel de evidencia sobre uso de endoprótesis cubiertas autoexpandibles, pero también son de utilidad clips, drenajes pigtail y pegamentos de fibrina. Para estenosis las dilataciones han mostrado buenos índices de efectividad. El tratamiento para la hemorragia endoluminal deberá de ser con terapia dual endoscópica convencional.
\end{abstract}

Palabras clave: Complicaciones. Cirugía bariátrica. Endoscopia terapéutica.

\section{Introducción}

Actualmente la obesidad es una pandemia. En México se ha estimado que la prevalencia de sobrepeso y obesidad es mayor en relación con la del promedio mundial; se estima que afecta a más del $75 \%$ de la población adulta y el $35 \%$ de la población infantil'. La cirugía bariátrica es el método más efectivo en el tratamiento de la obesidad mórbida. Así también, trata las comorbilidades asociadas a obesidad como diabetes, hipertensión arterial sistémica, hígado graso, síndrome de apnea obstructiva del sueño, dislipidemia, entre otras, coadyuvando en el tratamiento integral de estos pacientes. De esta manera el crecimiento en el número de procedimientos bariátricos realizados mundialmente se ha incrementado de manera importante, y a pesar de que actualmente la cirugía bariátrica es de baja morbilidad y mortalidad, el número total de complicaciones se ha incrementado. Las cirugías más realizadas mundialmente son la manga gástrica (MG), bypass gástrico en $Y$ de Roux (BGYR), bypass gástrico de una anastomosis (BGUA), SADI-S; y aunque no se realiza comúnmente en la actualidad, persisten una gran cantidad de pacientes portadores de banda gástrica ajustable (BG). Existen múltiples clasificaciones para las complicaciones poscirugía bariátrica. Las más importantes por su relevancia en cuanto a mortalidad y morbilidad asociada son: fugas o fístulas de anastomosis o líneas de grapas, estenosis o torsiones y hemorragia. Además, estas complicaciones serán susceptibles de tratamiento endoscópico. En la tabla 1 se señalan la frecuencia de las principales complicaciones susceptibles de tratamiento endoscópico de acuerdo con los principales procedimientos.

\section{Fugas o fístulas poscirugía bariátrica}

De acuerdo con el tiempo de aparición, las fístulas se clasifican en agudas cuando se presentan los primeros 7 días, tempranas 1-6 semanas, tardías de 6 a 12 semanas y crónicas posterior a 12 semanas $^{8}$. Las

\section{Correspondencia:}

${ }^{*}$ Carlos Valenzuela-Salazar

E-mail: carlosvalenzuelas@gmail.com 
Tabla 1. Frecuencia de las principales complicaciones susceptibles de tratamiento endoscópico de acuerdo con los principales procedimientos

\begin{tabular}{|l|l|c|l|c|}
\hline & \multicolumn{2}{|c|}{ Tempranas (\%) } & \multicolumn{2}{c|}{ Tardías (\%) } \\
\hline $\begin{array}{l}\text { Manga } \\
\text { gástrica }\end{array}$ & Fuga & $2.2-2.4$ & Fuga & 2.4 \\
\hline & Sangrado & $0-8.7$ & Estenosis & $0.69-2$ \\
\hline $\begin{array}{l}\text { Bypass } \\
\text { en Y } \\
\text { Roux-7 }\end{array}$ & Fuga & $0.6-4.4$ & $\begin{array}{l}\text { Estenosis de } \\
\text { anastomosis }\end{array}$ & $3-28$ \\
\hline & Sangrado & 1.15 & $\begin{array}{l}\text { Sangrado } \\
\text { crónico } \\
\text { Úlcera marginal }\end{array}$ & $0.6-25$ \\
\hline
\end{tabular}

fístulas poscirugía bariátrica pueden derivar en altas tasas de morbilidad, reintervenciones y mortalidad.

A la par de la ayuda de los tratamientos endoscópicos para esta patología, debemos asegurarnos del adecuado control de la sepsis y drenaje pertinente de abscesos o colecciones intraabdominales del paciente complicado. Uno de los tratamientos endoscópicos más exitosos en la resolución de esta patología es la colocación de prótesis metálicas autoexpandibles cubiertas. Estas ofrecen la ventaja de brindar una ferulización para la cicatrización de la fístula, así como mantener el lumen permeable, lo que permite la alimentación o el paso de sonda naso enteral. En revisiones sistemáticas y metaanálisis clásicos se ha observado un índice de resolución en fístulas posmanga gástrica del $72.8 \%$ y para bypass gástrico en $Y$ del $76.1 \%$, dejándose entre 40 a 50 días en estos estudios a pesar de que el tiempo recomendado es de entre 6 y 8 semanas $^{9}$. Una revisión sistemática y metaanálisis publicada en 2021 que incluyó 40 estudios con 493 pacientes, demostró un índice global de cierre de fístulas utilizando prótesis del $92 \%$, cierre con clips Montados (OTSC) con éxito del $67.1 \%$ y con pegamento de fibrina de entre 92 y $100 \%$. La frecuencia de migración de prótesis en este estudio fue de hasta $23 \%{ }^{10}$. Las complicaciones reportadas más frecuentemente son migración, síntomas como saciedad temprana, náusea y dolor. Una alternativa en el tratamiento de las fugas agudas y tempranas es el uso de drenaje interno mediante uso de catéteres cola de cochino (pigtail). El fundamento de esta práctica es favorecer el drenaje de la cavidad de la fístula hacia el interior y no hacia la cavidad o la piel. En una revisión sistemática del 2019 en la que se incluyeron 11 estudios retrospectivos con un total de 681 pacientes de fuga posmanga el uso de doble catéter pigtail mostró tasas de éxito del $84.7 \%$. Las complicaciones reportadas de este tratamiento fueron hemorragia, migración del drenaje y estenosis ${ }^{11}$. Una alternativa de drenaje interno es mediante uso de presión negativa (e-vac). Si bien el principio es similar al drenaje interno propuesto por los pigtails, la ventaja de este sistema es la aspiración continua, que favorece tejido de granulación. Se han realizado estudios en diversos tipos de cirugías del tracto gastrointestinal alto y hay poca evidencia en pacientes operados de cirugía bariátrica. Sin embargo, en estudios publicados en 2020 la tasa de éxito es superior al $90 \%{ }^{12}$. El uso de clips montados sobre el endoscopio (OTSC), ha sido utilizado con éxito también en el cierre de fístulas como terapia única o combinada. En los estudios más recientes del tipo revisión sistemática y metaanálisis de estudios retrospectivos, mostró ser efectivo en fístulas poscirugía bariátrica tanto de manga como de bypass mostrando tasas de éxito del $67.1 \%$ como terapia única. La evidencia del uso de sutura endoscópica para el cierre de fístulas continúa siendo escasa con tasas de éxito moderadas y alta tasa de recurrencia. Especialmente útiles en casos de fístulas pequeñas con agudas o tempranas sin estudios recientes para comentar. Sin embargo, los pegamentos tisulares como monoterapia o combinada para la misma finalidad ha mostrado mayor utilidad, a pesar de requerirse en ocasiones múltiples aplicaciones. No obstante que la evidencia reciente se limita a series de casos, las tasas de éxito de aplicación repetitiva se han reportado superior al $90 \%{ }^{10}$.

\section{Estenosis poscirugía bariátrica}

Complicación que se puede presentar en cualquier paciente operado de cirugía bariátrica y que oscila entre 2 y el $28 \%$ en gastroyeyunoanastomosis de bypass y entre 0.1 y $4 \%$ posmanga. Suelen ser ocasionadas por falla técnica en las fases tempranas 0 multifactoriales en las tardías (> 45 días). El estándar en el tratamiento de las estenosis de la GYA postBGYR es mediante balones de dilatación hidroneumáticos llevando las estenosis a partir de $8 \mathrm{~mm}$, cada 2 a 4 semanas, hasta $18 \mathrm{~mm}$ como diámetro máximo y con un objetivo meta de $15 \mathrm{~mm}$. Las tasas de éxito reportadas en estudios clásicos oscilan entre el 77 y el $100 \%{ }^{13}$. A diferencia de estas, las estenosis en manga gástrica suelen ser mecánicas por estrechez comúnmente en sitio de incisura angular, o bien funcionales debido a una torción del tubo gástrico. El tratamiento es mediante uso de balones de dilatación 
de acalasia de entre 30 a $40 \mathrm{~mm}$. En una revisión sistemática publicada en 2020 se reportó una resolución endoscópica de hasta el $82 \%$ de los casos, siendo el uso de balones de dilatación de acalasia la mejor opción terapéutica. No existen estudios recientes de mayor nivel de evidencia sobre el tratamiento endoscópico de estenosis poscirugía bariátrica, pero el tratamiento endoscópico continúa siendo el de primera línea para el tratamiento de esta complicación.

\section{Hemorragia poscirugía bariátrica}

La incidencia de hemorragia intraluminal oscila globalmente entre el 2 y $4 \%$. Si bien la mayor parte se autolimita, se deberá intentar manejo endoscópico ante una hemorragia persistente 0 intensa. Los métodos estándar de hemostasia endoscópica aplican para estos pacientes como los son la terapia dual con epinefrina, clips, térmicos y polvos. En úlceras marginales se debe aplicar tratamiento con IBP, erradicación de Helicobacter pylori cuando aplique y retiro de material de sutura. Si la hemorragia no es accesible a endoscopia convencional debido a provenir del estómago excluido en BGYR o de la anastomosis yeyunoyeyunal, se deberán intentar el control mediante enteroscopia retrógrada o exploración quirúrgica. No existe evidencia reciente que comentar sobre el tratamiento endoscópico de hemorragia poscirugía bariátrica.

\section{Financiamiento}

La presente investigación no ha recibido ayudas específicas provenientes de agencias del sector público, sector comercial o entidades sin ánimo de lucro.

\section{Conflicto de intereses}

Los autores declaran que no hay conflicto de intereses.

\section{Bibliografía}

1. Instituto Nacional de Estadística y Geografía. Encuesta Nacional de Salud y Nutrición 2018. Diseño muestral [Internet]. México: INEGI; 2018. Disponible en: https://www.inegi.org.mx/contenidos/programas/ensanut/2018/doc/ensanut_2018_diseno_muestral.pdf

2. García-García ML, Martín-Lorenzo JG, Ramón Lirón-Ruiz R, Torralba-Martínez JA, García-López JA, Aguayo-Albasini JL. Perioperative complications following bariatric surgery according to the Clavien-Dindo classification. Score validation, literature review and results in a single-centre series. Surg Obes Relat Dis. 2017;13(9):1555-61.

3. Chang SH, Stoll CRT, Song J, Varela JE, Eagon CJ, Colditz GA. The effectiveness and risks of bariatric surgery. An updated systematic review and meta-analysis, 2003-2012. JAMA Surg. 2014;149(3):275-87.

4. Puzziferri N, Roshek III TB, Mayo HG, Gallagher R, Belle $\mathrm{SH}$ Livingston $\mathrm{EH}$. Long- term follow-up after bariatric surgery: A systematic review. JAMA. 2014;312(9):934-42.

5. Ma It T, Madura $2^{\text {nd }} \mathrm{JA}$. Gastrointestinal complications after bariatric surgery. Gastroenterol Hepatol (N Y). 2015;11(8):526-35.

6. Gu L, Chen B, Du N, Fu R, Huang X, Mao F, et al. Relationship between bariatric surgery and gastroesophageal reflux disease: A systematic review and meta-analysis. Obes Surg. 2019;29(12):4105-13.

7. Cai JX, Schweitzer MA, Kumbhari V. Endoscopic management of bariatric surgery complications. Surg Laparosc Endosc Percutan Tech. 2016;26(2):93-101.

8. Rosenthal RJ, Aceves Diaz A, Arvidsson D, Baker RS, Basso N, Bellanger D, et al.; International Sleeve Gastrectomy Expert Panel. International Sleeve Gastrectomy Expert Panel, International Sleeve Gastrectomy Expert Panel Consensus Statement: best practice guidelines based on experience of $>12,000$ cases. Surg Obes Relat Dis. 2012;8(1):8-19.

9. Okazaki O, Bernardo W, Brunaldi V, de Clemente Junior CC, Minata MK, de Moura DT, et al. Efficacy and safety of stents in the treatment of fistula after bariatric surgery: A systematic review and meta-analysis. Obes Surg. 2018;28(6):1788-96.

10. Rogalski P, Swidnicka-Siergiejko A, Wasielica-Berger J, Zienkiewicz D, Wieckowska B, Wroblewski E, et al. Endoscopic management of leaks and fistulas after bariatric surgery: a systematic review and meta-analysis. Surg Endosc. 2021;35(3):1067-87.

11. Giuliani A, Romano L, Marchese M, Necozione S, Cianca G, Schietroma M, et al. Gastric leak after laparoscopic sleeve gastrectomy: management with endoscopic doublé pigtail drainage. A sistematic review. Surg Obes Relat Dis. 2019;15:1414-9.

12. Sendino O, Loras C, Mata A, Momblán D. Eficacia y seguridad de la terapia de vacío endoscópica para el tratamiento de perforaciones y dehisencias anastomóticas del tracto digestivo superior. Gastroenterol Hepatol. 2020:43(8):431-8.

13. Eisendrath $P$, Deviere J. Major complications of bariatric surgery: endoscopy as first-line treatment. Nat Rev Gastroenterol Hepatol. 2015;12:701-71. 